\title{
Evaluation of the neutrophil:lymphocyte ratio as an indicator of chronic distress in the laboratory
} mouse

\section{Debra L Hickman}

When evaluating the effect of husbandry and biomethodologies on the well-being of laboratory mice, it is critical to utilize measurements that allow the distinguishing of acute stress from chronic stress. One of the most common measurements of stress in laboratory animals is the corticosterone assessment. However, while this measurement provides a highly accurate reflection of the animal's response to acute stressors, its interpretation is more prone to error when evaluating the effect of chronic stress. This study evaluated the use of the neutrophil:lymphocyte (NE:LY) ratio as an assessment of chronic stress in male and female $\mathrm{C} 57 \mathrm{Bl} / 6 \mathrm{~N}$ mice as compared to serum corticosterone. One group of mice was exposed to mild daily stressors for 7 days, while the control group was handled with normal husbandry. The NE:LY ratio and serum corticosterone levels were significantly elevated in the chronically stressed mice, though a significant increase in corticosterone was only significant in males when compared by sex. The chronically stressed mice also demonstrated significantly fewer entries into the open arms and less time spent in the open arms of the elevated plus maze, suggesting that the mild daily stressors had induced a state of distress. The findings of this study confirm that the NE:LY ratio is a valid measurement for chronic stress in the laboratory mouse. However, these assays do not distinguish between distress or eustress, so behavioral and physiological assessments should always be included to determine a complete assessment of the well-being of the mouse.

When designing studies that use animal models, it has been recognized that the results are more translatable to human disease when the subjects are healthy with minimal stressors ${ }^{1-6}$. But, not all stress is bad, so it is critical that evaluations of animal well-being differentiate between distress (that which results in decreases in animal well-being as the animal is unable to cope or adapt) and eustress (stressors which enhance the functioning of the animal) ${ }^{7,8}$. Appropriate selection of assessments of animal well-being can assist laboratory animal professionals as they seek to evaluate and improve current practices ${ }^{9}$, especially with mice.

Generally, the most common method to assess laboratory mouse well-being has been focused on assessment of the response to acute stressors (i.e. an injection, brief restraint, a cage change ${ }^{10}$. These stress events have consistently been associated with elevations in mouse serum and fecal corticosterone $e^{6,11,12}$ and neuropeptides ${ }^{13}$. Mice that have been exposed to acute stressors also have shown increased anxiety when assessed by the elevated plus maze and other behavioral assessments ${ }^{14-17}$, in addition to elevations of heart rate and blood pressure ${ }^{18-20}$.

It has been more challenging to measure states of chronic stress, where an animal was exposed to the stressors for hours or days instead of seconds or minutes. Corticosteroids are commonly used in these assessments, but interpretation is complicated because corticosterone levels are influenced by factors that include the method of sample collection, sex, immune status, circadian rhythm, and nutritional status ${ }^{10}$. They also increase when an animal experiences extreme fear (distress) or extreme pleasure (eustress) ${ }^{21}$, requiring the addition of behavioral assessment to accurately determine the state of the animal. Changes in body weight and organ weights relative to body weight have been among the more reliable measures of chronic stress as these measure change more slowly, making them a stronger reflection of the effects of exposure to chronic stressors ${ }^{22-24}$.

Work by this laboratory and others have noted that chronic stressors that induce increase glucocorticoid responses also induce 
an apparent immunosuppression that presents within a couple of hours of elevated corticosterone exposure as a neutrophilia that may include a concurrent lymphopenia ${ }^{25-28}$. These immunologic changes are also seen in disorders which are associated with elevated glucocorticoids ${ }^{29,30}$ and other chronic illnesses, supporting the role of glucocorticoids in the apparent immunosuppression. The high correlation coupled with the relatively low cost to obtain a complete blood count has resulted in use of the neutrophil:lymphocyte (NE:LY) ratio to assess stress in multiple species ${ }^{25,27}$.

The objective of this study was to determine if the NE:LY ratio was consistent with serum corticosterone as a measurement of chronic stress in the laboratory mouse. Additionally, we included the elevated plus maze to determine if the NE:LY ratio accurately measured distress in the chronically stressed mice, as compared to the behavior of the non-manipulated, non-stressed mice.

\section{MATERIALS AND METHODS}

\section{Ethical statement}

The Indiana University School of Medicine (IUSM) animal care and use program was in compliance with federal regulations and accredited by AAALAC, International. All procedures were approved by the IUSM IACUC prior to initiation of the study.

\section{Experimental design}

The study was structured with a between-subjects design where subjects were randomly assigned to the independent variable of "Stress Exposure" which had two groups. The first group ("chronically stressed") was exposed to random, mild daily stressors for 7 consecutive days. The second group ("non-stressed") was housed and handled as per usual in the animal facility. Each group consisted of 6 males and 6 females which were randomly assigned to their treatment group upon arrival at the animal facility. Random assignment was accomplished by assigning each animal a number, then using a random number generator to assign animals to treatment groups.

\section{Animals}

Twelve male and twelve female C657BL/6NCrl mice (Charles River Laboratories, Wilmington, MA) were used for this study. The mice were approximately 10 weeks of age. As social housing in mice has been demonstrated to prevent stress-associated responses ${ }^{31,32}$, the mice in the chronically stressed group were housed individually, while the non-stressed animals were group housed. They were allowed to acclimate for 7 days prior to initiating the experimental manipulations.

All animals were housed in shoebox caging with filter tops (Alternative Design, Siloam Springs, AR) on corncob bedding (Bed O' Cob, Andersons, Maumee, $\mathrm{OH}$ ) under static conditions. All mice were provided with tissue material for nesting. Both males and females were housed in the same room, but the mice in each treatment group were housed in different rooms as described below. The cages measured $18.5 \mathrm{~cm} \mathrm{~W} \mathrm{x} 30 \mathrm{~cm} \mathrm{D} \mathrm{x} 12 \mathrm{~cm} \mathrm{H}$. Feed (LabDiet 2018, St. Louis, MO) and water were provided ad libitium. Standard operating procedures for the animal facilities required that all cages be changed at least once weekly. Soiled cages were sanitized in a mechanical cage washer with a final rinse temperature of $180^{\circ} \mathrm{F}\left(82^{\circ} \mathrm{C}\right)$ and autoclaved prior to reuse. The macroenvironment was kept on a 12:12-h light:dark cycle (lights on at 0:700, off at 19:00) with temperature and humidity maintained at $72^{\circ} \mathrm{F}$ $\left(22^{\circ} \mathrm{C}\right)$ and at least $30 \%$, respectively. Indirect sentinels were utilized to assess colony health. At the time of this study, the colony was free of mouse parvovirus, mouse coronavirus, mouse theliovirus, pneumonia virus of mice, Sendai virus, Reovirus 3, mouse rotavirus, lymphocytic choriomeningitis virus, Mycoplasma pulmois, and ectoparasite and endoparasites.

\section{Chronic stress}

Half of the mice were exposed to a daily stressor for 7 consecutive days. These stressors were presented in a random order and included: (1) forced swim, (2) heat stress, (3) shaker stress, and (4) restraint stress modified from previously published work ${ }^{33}$. For the forced swim, the mouse was gently placed in a $13 \mathrm{~cm}$ diameter x $30 \mathrm{~cm}$ tall clear plexiglass cylinder filled with water at $70^{\circ}$ to $75^{\circ} \mathrm{F}\left(21^{\circ}\right.$ to $\left.24^{\circ} \mathrm{C}\right)$. They remained in the water until they began floating with positional adjustments or 2 minutes, whichever was longer. They were removed, dried and allowed to warm. After 10 minutes of rest, the swim stress was repeated for a maximum of 4 sessions per mouse. For the heat stress, the mouse was placed in a clean cage with no bedding and a wire mesh to prevent escape. An incandescent light was positioned above the cage to create heat in the cage. The temperature was monitored and the target temperature was $85^{\circ} \mathrm{F}\left(29^{\circ} \mathrm{C}\right)$. The mouse was removed if the intracage temperature exceeded $90^{\circ} \mathrm{F}\left(32^{\circ} \mathrm{C}\right)$. They stayed in the cage for 2 hours and were continuously monitored during this stressor. For the shaker stress, the mouse remained in its home cage which was placed on a plate shaker that provided gentle, continuous shaking at no more than $160 \mathrm{rpm}$ for 2 hours. Mice were assessed every 30 minutes during this stressor. For the restraint stress, mice were restrained in a $50 \mathrm{ml}$ centrifuge tube with air-holes for a maximum of 3 hours. Mice were continuously monitored through this stressor. The actual times and stressor assignments are provided as Supplemental Data.

These mice were individually housed and held in a room isolated from other mice and outside of the main animal facility to minimize the potential effect of pheromones on other ongoing studies, including the non-stressed controls for this study. The cages for mice in this group were situated so that they were at average human eye level. These mice were tested and euthanized on day 8 of the study.

\section{Handling of non-stressed mice}

The non-stressed mice were housed in groups of 3 mice of the same sex per cage. They were handled normally, with no additional interactions with the research staff. The cages for mice in this group were situated so that they were at average human thigh to knee level. The non-stressed mice were tested and euthanized on day 7 of the study to avoid release of pheromones from the stressed mice influencing the response of the non-stressed mice.

\section{Elevated plus maze}

All mice were assessed on an elevated plus maze at the end of the 7 consecutive days of daily stressors for the chronically stressed 
mice. The maze measured $25 \mathrm{~cm} \mathrm{~L} \mathrm{x} 5 \mathrm{~cm} \mathrm{~W}$ open arms, $25 \mathrm{~cm} \mathrm{~L} \mathrm{x}$ $5 \mathrm{~cm} \mathrm{~W} \mathrm{x} 16 \mathrm{~cm} \mathrm{H}$ closed arms, center platform of $5 \mathrm{~cm} \times 5 \mathrm{~cm}$. The closed arms were opaque. Mice were brought to the testing room and allowed a minimum of 30 minutes to acclimate to the training room before being tested.

Each mouse was placed on the center platform to start the EPM and digitally recorded for 10 minutes. At the end of 10 minutes, the mouse was removed from the maze and the maze was cleaned with $70 \%$ isopropyl alcohol before the next mouse. The proportion of entries into the open arms relative to the total number of arm entries was compared between treatment groups. The proportion of time each mouse spent in the open or closed arms relative to the total time in the maze was also compared between treatment groups. Each mouse was tested once. All trials were between 8:00 and 12:00. All non-stressed mice were tested separately from the chronically stressed mice. Data from two non-stressed and one chronically stressed mouse were not collected due to complications with the digital recording.

\section{Blood collection}

After being tested on the elevated plus maze, each mouse was anesthetized with pentobarbital $(390 \mathrm{mg} / \mathrm{ml}$, Fatal Plus, Vortech Pharmaceuticals, Dearborn, MI) administered IP. Once anesthetized, a terminal blood collection was collected from the heart. Half of the sample was placed in an EDTA treated tube and the remainder was placed in a serum separator tube and subsequently centrifuged to allow collection of the serum. The serum samples were stored at $-80^{\circ} \mathrm{F}\left(-62^{\circ} \mathrm{C}\right)$ until processed. All samples were collected between 8:00 and 12:00 to control for alterations due to circadian rhythm.

\section{Hematology}

The EDTA sample was placed in a Hemavet (Drew Scientific, Waterbury, CT) and a complete blood count was performed. The mean of the white blood count, total number of neutrophils, total number of lymphocytes, and NE:LY ratio were compared between treatment groups.

\section{Serum corticosterone assay}

The serum corticosterone levels were assessed using a commercially available ELISA kit (Corticosterone rat/mouse, MP Biomedicals,
LLP, Solon, OH). The mean serum corticosterone levels were compared between treatment groups. We were unable to collect enough blood for serum from 2 of the non-stressed mice.

\section{Statistical analysis}

To determine the appropriate animal numbers to be used prior to the initiation of the study, we performed a power analysis using our previous work with the neutrophil:lymphocyte ratio in our laboratory as the basis for the analysis. An online calculator was utilized ${ }^{34}$ with $\mu_{\mathrm{A}}$ set at $0.4, \mu_{\mathrm{B}}$ set at $0.25, \sigma$ set at 0.09 , a sampling ratio (ê) of 1 , power at 0.80 and $\alpha$ at $5 \%$. This calculated a sample size of 6 animals per sex per group.

Data analyses were performed using a generalized linear model (JMP 10, Cary, NC). The animal was the experimental unit. A single observation was made for each animal and this data was averaged per animal for analysis using a simple model of Treatment, Sex, and their interaction. If interactions were found to be non-significant, the models were updated by removing the interactions terms to retest the main effects and these values were reported. A full-factorial GLM was completed for each observational unit. If interactions were found to be significant, these results were reported. All summary data are presented as box-and-whisker plots with whiskers representing min and max values, boxes first and third quartiles, and middle line the median value.

\section{RESULTS}

\section{Elevated plus maze}

The non-stressed mice spent a significantly higher proportion of their 10 minutes in the open arms of the elevated plus maze as compared to the chronically stressed mice $(\mathrm{F}[1,20]=8.4119, p=0.0088)$ (Fig. 1a; see Supplementary Figs. 1-5 for raw data plots). There were no significant differences in the proportion of time spent in the closed arms between the non-stressed and the chronically stressed mice $(F[1,20]=0.0433, p=0.8372)$ (Fig. 1b; see Supplementary Figs. 1-5 for raw data plots). The proportion of entries into the open arms of the elevated maze was significantly higher for the non-stressed mice as compared to the chronically stressed mice $(\mathrm{F}[1,20]=9.3154, p=0.0063)$ (Fig. 1c; see Supplementary Figs. 1-5 for raw data plots). However, there were no significant differences
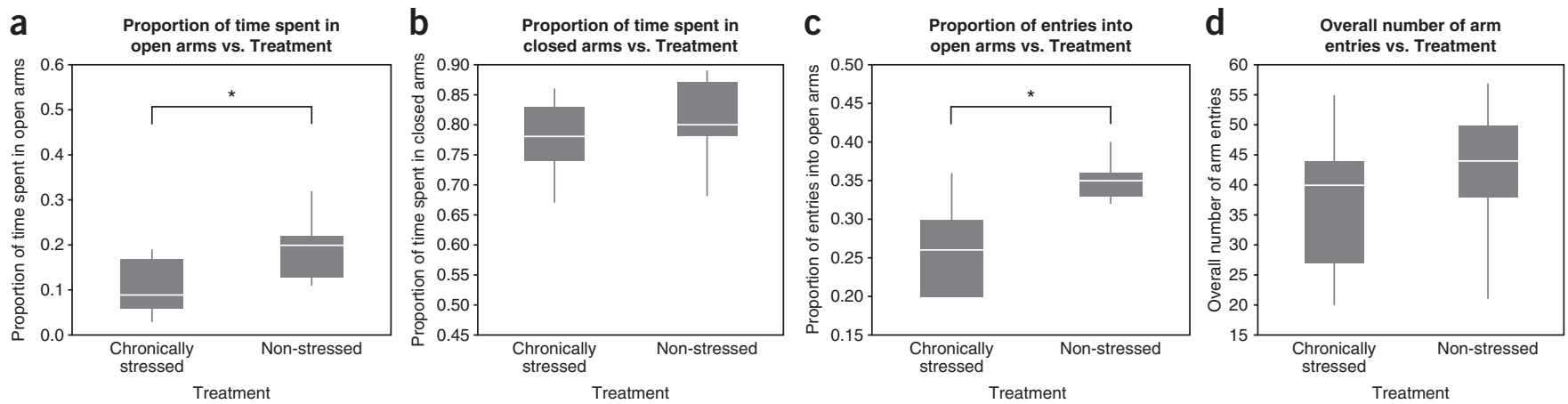

FIGURE 1 | Results of the elevated plus maze behavioral assay. (a) Box-and-whisker plot with solid white line representing the median proportion of time spent in the open arms. (b) Box-and-whisker plot with solid white line representing the median proportion of time spent in the closed arms. (c) Box-and-whisker plot with solid white line representing the median proportion of entries into the open arms. (d) Box-and-whisker plot with solid white line representing the median total number of entries into all arms. Significance is set at $p<0.05$ and is indicated by *. 

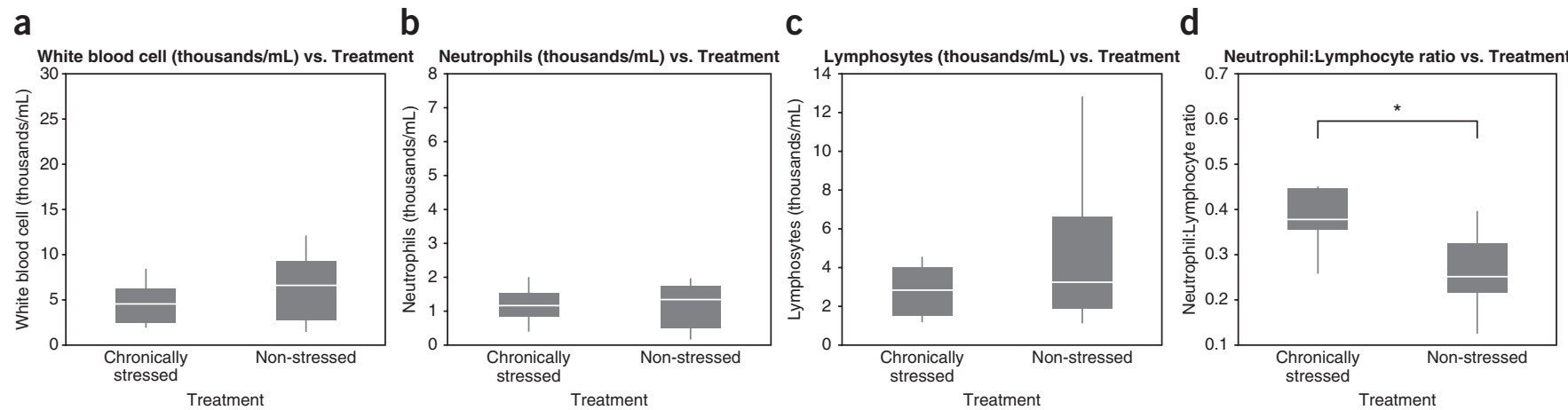

FIGURE 2 | Results of the hematology assay. (a) Box-and-whisker plot with solid white line representing the median total white blood cell count (thousand/mL). (b) Box-and-whisker plot with solid white line representing the median total neutrophils (thousand/mL). (c) Box-and-whisker plot with solid white line representing the median total lymphocytes (thousands/mL). (d) Box-and-whisker plot with solid white line representing the median NE:LY ratio. Significance is set at $p<0.05$ and is indicated by *.

in overall number of arm entries between the non-stressed and the chronically stressed mice $(\mathrm{F}[1.20]=1.8778, p=0.1858)$ (Fig. 1d; see Supplementary Figs. 1-5 for raw data plots). There was no interaction of sex with treatment for the proportion of time spent in the open arms $(p=0.0695)$, for the proportion of time spent in the closed arms $(p=0.1443)$, for the proportion of entries into the open arms $(p=0.7391)$, and for the total number of arm entries $(p=0.8254)$.

\section{Hematology}

There were no significant differences between the treatment groups in the total white blood cell count $(\mathrm{F}[1,23]=2.0245, p=0.1682)$ (Fig. 2a; see Supplementary Figs. 6-9 for raw data plots), the total number of neutrophils $(\mathrm{F}[1,23]=0.5778, p=0.4549)$ (Fig. 2b; see Supplementary Figs. 6-9 for raw data plots), or the total number of lymphocytes $(\mathrm{F}[1,23]=3.4353, p=0.0767)$ (Fig. 2c; see Supplementary Figs. 6-9 for raw data plots). The NE:LY ratio of the chronically stressed mice was significantly increased as compared to the non-stressed mice $(F[1,23]=7.4200, p=0.0121)$ (Fig. 2d; see Supplementary Figs. 6-9 for raw data plots). There was no interaction of sex with treatment for the total white blood cell count ( $p=0.8018)$, total number of neutrophils $(p=0.6877)$, total number of lymphocytes $(p=0.8665)$, and the NE:LY ratio $(p=0.5542)$.

\section{Corticosterone}

The chronically stressed mice exhibited a significant increase in their levels of serum corticosterone as compared to the non-stressed mice $(\mathrm{F}[1,21=5.3431, p=0.0310)$. There was a significant interaction of sex and treatment $(p=0.0389)$. Chronically stressed male mice expressed significantly higher levels of serum corticosterone as compared to the non-stressed male mice $(F[1,8]=9.0825, p=0.0167)$, but females expressed no significant differences between non-stressed and chronically stressed mice $(\mathrm{F}[1,11]=0.1282, p=0.7270)$ (Fig. 3; see Supplementary Figs. 10 and 11 for raw data plots).

\section{DISCUSSION}

The mice that had been exposed to 7 days of mild stressors mice demonstrated a significant increase in their serum corticosterone levels, suggesting that these mice were more stressed as compared to the mice that were handled routinely. Not unexpectedly, the study also documented a sex difference in serum corticosterone levels, with the chronically stressed male mice exhibiting a significantly higher corticosterone level as compared to the chronically stressed female mice. Additionally, the behavioral assessment of the chronically stressed mice demonstrated that they were less likely to spend time on the open arms and less likely to enter the open arms of the elevated plus maze. As demonstrated by previous studies, these findings suggest that the mice were more anxious and distressed ${ }^{35}$ as compared to the mice that were handled routinely. These behavioral findings suggest that the daily stressors were adequately disturbing to create a situation of distress for these mice.

The finding that the NE:LY ratio was also increased in the chronically stressed mice suggests that this assessment can be a reliable tool for the measurement of stress in the laboratory mouse. There was also a non-significant neutrophilia and lymphopenia in the chronically stressed mice, but all values were within published normal for $\mathrm{C} 57 \mathrm{BL} / 6 \mathrm{NCrl}$ mice ${ }^{36}$. These findings are consistent with other published studies that chronic exposure to elevated corticosterone causes a shift of neutrophilia with or without a concurrent lymphopenia ${ }^{10,25,27}$, and can be measured successfully by evaluating the NE:LY ratio of experimental treatment groups as compared

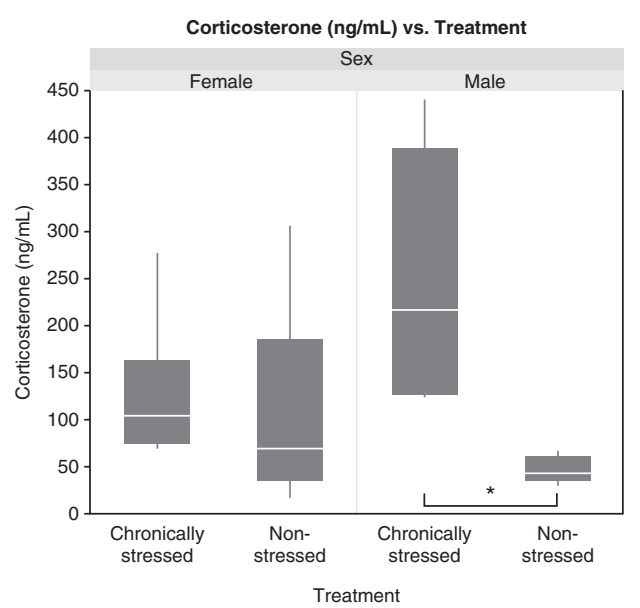

FIGURE 3 | Results of the corticosterone assessment $(\mathrm{ng} / \mathrm{mL})$. Significance is set at $p<0.05$ and is indicated by *. 
to control groups ${ }^{25,27}$. Because the differences were robust and reflective of the behavioral changes, this suggests that the NE:LY ratio is an appropriate measurement to use when assessing chronic distress in a clinically healthy mouse.

The measurement of the NE:LY ratio also has significant advantages over the measurement of serum corticosterone. Although this study utilized an automated hemocytology machine, the NE:LY ratio can be calculated from a blood smear by manually performing a differential ${ }^{25,27}$. Measurement of serum corticosterone requires the use of specialized kits and additional machinery, such as ELISA readers, for accurate calculation of the concentrations. As previously reported in the laboratory rat, the NE:LY ratio also is not influenced by the response to acute stressors, such as handling to obtain the blood collection ${ }^{27}$. It is also less sensitive to potentially confounding variables such as time of day, sex (which was seen in the serum corticosterone results of this study) and appetence, though it can be affected by chronic pathogen exposure ${ }^{25,27}$.

In this study, we conclude that the NE:LY ratio is an acceptable immunological measurement of exposure to chronic stress in the clinically healthy laboratory mouse. It can be run inexpensively without specialized equipment and is reliably robust in the face of individual and environmental factors. However, as should be considered for all assessments of stress in animals, this method should not be used alone, but as part of a multi-faceted panel of assessments that include physiologic and behavioral assessments to determine if changes are due to distress, eustress, or disease status ${ }^{9,10}$.

Note: Any Supplementary Information and Source Data files are available in the online version of the paper.

\section{ACKNOWLEDGMENTS}

This study was funded from internal sources. The author thanks Melissa Swan, Jessica Peveler, and Brittany Baker for their assistance with the data collection for this study.

\section{Competing Financial Interests}

The author declares no competing financial interests.

\section{Received 16 December 2016; accepted 11 April 2017}

Published online at www.nature.com/laban

1. Gouveia, K. \& Hurst, J.L. Reducing mouse anxiety during handling: effect of experience with handling tunnels. PLoS One 8, e66401 (2013).

2. Jackson, E. et al. Aspen shaving versus chip bedding: effects on breeding and behavior. Lab. Anim. 49, 46-56 (2015).

3. Miller, A.L. \& Leach, M.C. The effect of handling method on the mouse grimace scale in two strains of laboratory mice. Lab. Anim. 50, 305-307 (2016).

4. Pasalic, I. et al. Cage enrichment with paper tissue, but not plastic tunnels, increases variability in mouse model of asthma. Lab. Anim. 45, 121-123 (2011).

5. Poole, T. Happy animals make good science. Lab. Anim. 31, 116-124 (1997).

6. Rosenbaum, M.D., VandeWoude, S. \& Johnson, T.E. Effects of cage-change frequency and bedding volume on mice and their microenvironment. J. Am. Assoc. Lab. Anim. Sci. 48, 763-773 (2009).

7. Selye, H. Stress and distress. Compr. Ther. 1, 9-13 (1975).

8. Stern, C.M. Corticotropin-releasing factor in the hippocampus: eustress or distress? J. Neurosci. 31, 1935-1936 (2011).

9. Pekow, C. Defining, measuring, and interpreting stress in laboratory animals. Contemp. Top. Lab. Anim. Sci. 44, 41-45 (2005).
10. Broom, D. \& Fraser, A. Domestic Animal Behaviour and Welfare, 4th Ed. 58-69 (CAB International, 2010)

11. Zimprich, A. et al. A robust and reliable non-invasive test for stress responsivity in mice. Front. Behav. Neurosci. 8, 125 (2014).

12. Fitzpatrick, F. et al. Glucocorticoids in the nonobese diabetic (NOD) mouse: basal serum levels, effect of endocrine manipulation and immobilization stress. Life Sci. 50, 1063-1069 (1992).

13. Veissier, I. \& Boissy, A. Stress and welfare: two complimentary concepts that are intrinsically related to the animal's point of view. Physiology \& Behavior 92, 429-433 (2006).

14. Barden, N. et al. Antidepressant action of agomelatine (S 20098) in a transgenic mouse model. Prog. Neuropsychopharmacol. Biol. Psychiatry 29, 908-916 (2005).

15. Qin, M. \& Smith, C.B. Unaltered hormonal response to stress in a mouse model of fragile X syndrome. Psychoneuroendocrinology 33, 883-889 (2008).

16. Kedia, S. \& Chattarji, S. Marble burying as a test of the delayed anxiogenic effects of acute immobilisation stress in mice. J. Neurosci. Methods. 233, 150-154 (2014).

17. Swiergiel, A.H. \& Dunn, A.J. Feeding, exploratory, anxiety- and depression-related behaviors are not altered in interleukin-6-deficient male mice. Behav. Brain. Res. 171, 94-108 (2006).

18. Lee, D.L. et al. Hypertensive response to acute stress is attenuated in interleukin-6 knockout mice. Hypertension 44, 259-263 (2004).

19. Lee, D.L., Webb, R.C. \& Brands, M.W. Sympathetic and angiotensindependent hypertension during cage-switch stress in mice. Am. J. Physiol. Regul. Integr. Comp. Physiol. 287, R1394-1398 (2004).

20. van Bogaert, M.J. et al. Mouse strain differences in autonomic responses to stress. Genes Brain Behav. 5, 139-149 (2006).

21. Greenberg, N., Carr, J.A. \& Summers, C.H. Causes and consequences of stress. Integr. Comp. Biol. 42, 508-516 (2002).

22. Ullman-Cullere, M.H. \& Foltz, C.J. Body condition scoring: a rapid and accurate method for assessing health status in mice. Lab. Anim. Sci. 49, 319-323 (1999).

23. Pohorecky, L.A., Baumann, M.H. \& Benjamin, D. Effects of chronic social stress on neuroendocrine responsiveness to challenge with ethanol, dexamethasone and corticotropin-releasing hormone. Neuroendocrinology 80, 332-342. (2004).

24. Barsy, B., Leveleki, C., Zelena, D. \& Haller, J. The context specificity of anxiety responses induced by chronic psychosocial stress in rats: a shift from anxiety to social phobia? Stress 13, 230-237 (2010).

25. Davis, A., Maney, D. \& Maerz, J. The use of leukocyte profiles to measure stress in vertebrates: a review for ecologists. Funct Ecol 22, 760-772 (2008).

26. Cupps, T.R., Edgar, L.C. \& Fauci, A.S. Corticosteroid-induced modulation of immunoglobulin secretion by human B lymphocytes: potentiation of background mitogenic signals. J. Immunopharmacol. 4, 255-263 (1982).

27. Swan, M.P. \& Hickman, D.L. Evaluation of the neutrophil-lymphocyte ratio as a measure of distress in rats. Lab Anim. (NY) 43, 276-282 (2014).

28. Harris, J.G., Flower, R.J. \& Perretti, M. Endogenous corticosteroids mediate the neutrophilia caused by platelet-activating factor in the mouse. Eur. J. Pharmacol. 283, 9-18 (1995).

29. Kaufman, J. Diseases of the adrenal cortex of dogs and cats. Mod. Vet. Pract. 65, 429-434 (1984).

30. Munoz, M.C., Doreste, F., Ferrer, 0., Gonzalez, J. \& Montoya, J.A. Pergolide treatment for Cushing's syndrome in a horse. Vet. Rec. 139, 41-43 (1996).

31. Klein, B. et al. Activation of the mouse odorant receptor 37 subsystem coincides with a reduction of novel environment-induced activity within the paraventricular nucleus of the hypothalamus. Eur. J. Neurosci. 41, 793-801 (2015).

32. Guzman, Y.F. et al. Social modeling of conditioned fear in mice by nonfearful conspecifics. Behav. Brain. Res. 201, 173-178 (2009).

33. Gong, S. et al. Dynamics and correlation of serum cortisol and corticosterone under different physiological or stressful conditions in mice. PLoS One 10, e0117503 (2015).

34. HyLown Consulting, L. http://powerandsamplesize.com/Calculators/ Compare-2-Means/2-Sample-Equality.

35. Hogg, S. A review of the validity and variability of the elevated plus-maze as an animal model of anxiety. Pharmacol. Biochem. Behav. 54, 21-30 (1996).

36. Charles Rivers Laboratories. C57BL/6 Mouse Clinical Pathology Data http:// www.criver.com/files/pdfs/rms/c57bl6/rm_rm_r_c57bl6_mouse_clinical_ pathology_data (2016). 